\title{
Meshing strategy for hybrid tet-hex PHES layered bed heat storage
}

\author{
Jędrzej Mosiężny ${ }^{1 *}$, and Bartosz Ziegler ${ }^{1}$ \\ ${ }^{1}$ Poznań University of Technology, Chair of Thermal Engineering, Piotrowo 3, 60-965 Poznań, Poland
}

\begin{abstract}
Article describes a meshing strategy for efficient computation of generic layered heat storage device for pumped heat energy storage (PHES). Mesh for structural solid body and fluid boundary layer is with unstructured flow field is presented. A rationale for geometry choice, mesh topology and different types of mesh topologies considered in this study. The effect of coarse and fine mesh on computational time and accuracy of results for transient analysis of coupled fluid and heat flow is described.
\end{abstract}

\section{Nomenclature}

TES - Thermal Energy Storage

ES - Energy Storage

FES - Flywheel energy storage

CAES - Compressed air energy storage

PS - Power Storage

PSH - Pumped Storage Hydroelectricity

PHES - Pumped Heat Energy Storage

RE - Renewable Energy

\section{Background}

Rapid growth of global electrical power demand next to the climate change issue [1] is influenced by introduction of new environmental regulations concerning emission reductions of carbon dioxide [2]. This goal is realized by fast increase of renewable energy (RE) technologies share to energy mix of many countries, mostly solar and wind power. Utilization of these sources depends on weather conditions and potential of power generation is highly variable. Unproductive periods without wind and sunless days are interlaced with the peaks of electricity production exceeding the current demand. This surplus should be stored or otherwise is wasted, what causes rise of production costs as well as premature network wear.

Literature analysis has indicated that TES offers relatively high energy density and much lower price per $\mathrm{kWh}$ of built storage comparing to very high energy density batteries. Classical TES approach allows to obtain efficiencies up to $60 \%$. However such high value is very difficult to achieve and in case of low short term and long term energy prices fluctuations this value does not provide sufficient cost effectiveness. Therefore development of this technology is focused on investigations of round-trip efficiency (the ratio of energy put in to energy retrieved from storage) increasing concepts. Pumped heat energy storage is one of the most promising TES derived approach which has the potential to meet this demand [3]. PHES concept is based on heat pump principle of operation during loading and turbomachinery devices during discharging of hot side storage tank.

Small number of papers describe existing prototypes of PHES in the context of heat transfer and storage components of the system [4]. This paper concerns the mesh preparation for conjugate heat transfer CFD calculations of fluid and heat flow in fixed bed heat storage reservoir.

\section{Approach to geometry and meshing}

\subsection{Preparation of the geometry}

The study is performed on a segment of generic layered thermal storage as presented in Fig 1.

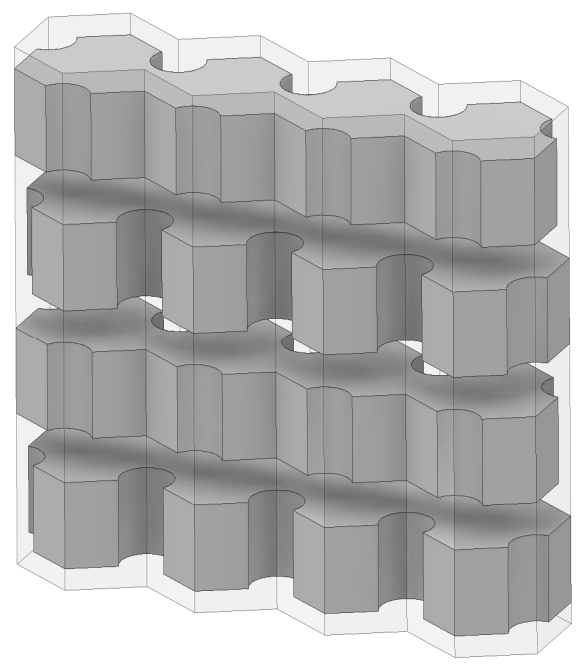

Fig. 1. Segment of layered thermal storage.

\footnotetext{
Corresponding author: jedrzej.mosiezny@put.poznan.pl
} 
The CAD geometry is composed of two solid body (in context of CAD modelling) regions representing layers of storage and fluid region respectively.

Individual holes in the discussed geometry are arranged in a triangular/hexagonal pattern and are shifted in relation to upper and lower level. Hole diameter is $40 \mathrm{~mm}$, distance between hole centrelines is $100 \mathrm{~mm}$, layer thickness is $50 \mathrm{~mm}$ and distance between layers is $25 \mathrm{~mm}$. Geometry does not represent any particular storage design (Fig. 2).

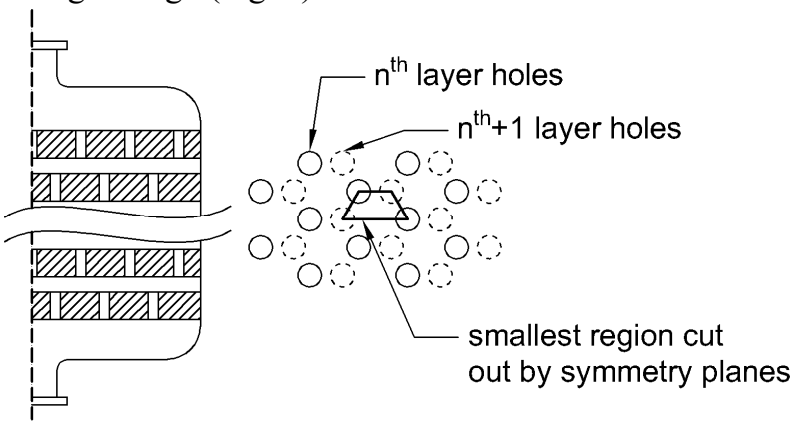

Fig. 2. Hole pattern overlapping.

It was found that the flow phenomena occurring in such entity are mostly in the direction of flow with minimal activity in radial, with regard to hole centrelines, direction. Therefore presented generic geometry was then sliced to obtain smallest possible volume that represents a single "cell" of the storage (Fig 3.). Side walls will be marked as symmetry boundary condition.

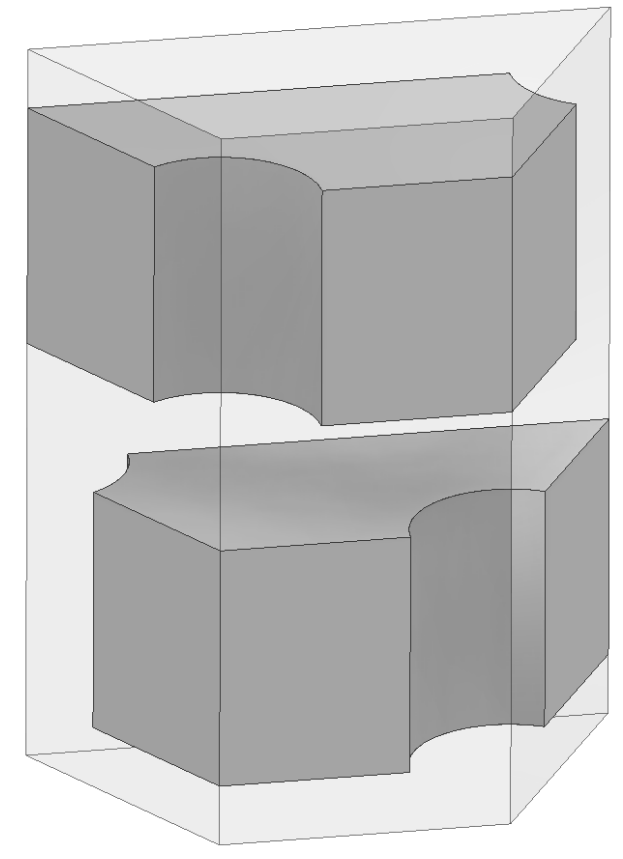

Fig. 3. Basic Geometry cell for CFD analysis.

\subsection{Meshing approach and topology}

Mesh was created in Pointwise V18.0R4 meshing software.

Based on previous experiences of the research team, a structural, hexahedral mesh was intended for this study. Although not very complex, the geometry does not mesh with fully hexagonal meshes without interfaces, therefore a following approach was made.

A topology for solid body (dark grey in Fig. 2) was created on the face of the solid body perpendicular to the flow direction (Fig 4). As the software used allows for "patch independent" meshing, that is: mesh information can be independent from the geometry, both solid and fluid regions were meshed during this operation.

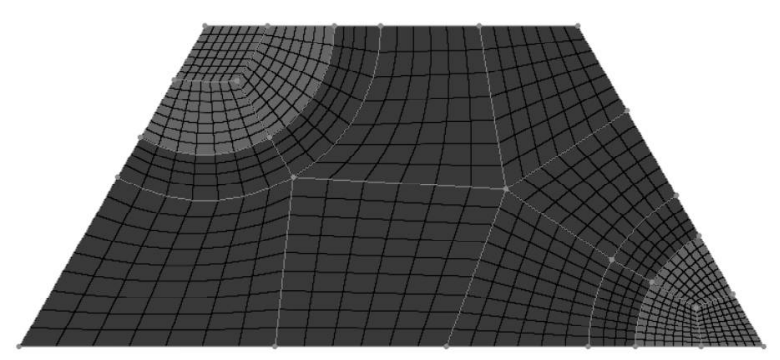

Fig. 4. Coarse mesh topology for solid region (dark grey) and fluid region (light gray)

The presented topology was then extruded in the direction of the flow to create the volume mesh for the solid body region and flowpath. Upper and lower surfaces were then extruded again to create a flow boundary layer. Such approach resulted in a typical htopology of the fluid boundary layer. Final solid region with fluid boundary region was mirrored and translated to form the second solid.

Remaining fluid volume was then filled with unstructured, isotropic tetrahedra with pyramid transitioning layer (Fig 5).

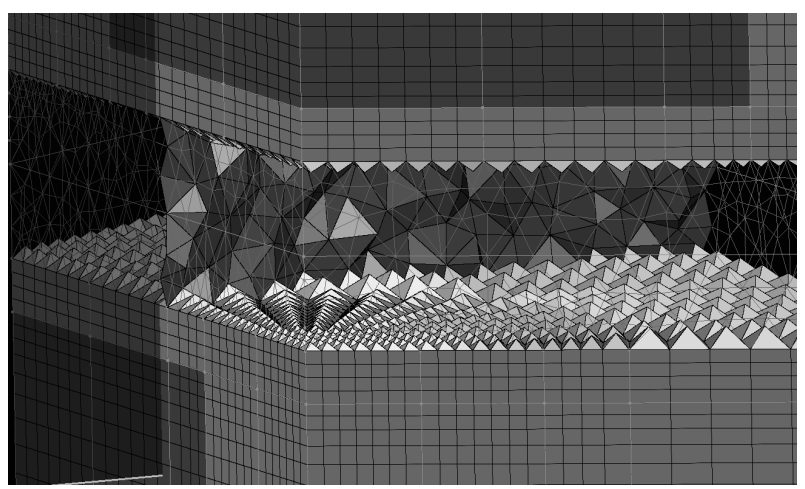

Fig. 5. Overview of mesh elements in coarse mesh; dark grey hexa: solid, light grey hexa: fluid BL, light grey pyramids: transition layer, dark grey tet: flowfield. 
Such basic geometry was then copied to form 32 solid domain layers (or 16 pairs with shifted hole patten) with one fluid domain around them.

One of the early approaches for meshing was to create an o-grid topology in the fluid boundary. Although the mesh quality in the fluid boundary was more than acceptable, the rounded section of the o-grid made it impossible to create a high quality pyramid transition layer and transition to tetrahedra.

Final mesh consists a 4 meter long cut from layered bed heat storage device, including $30 \mathrm{~cm}$ inlet and outlet regions. Such geometry represents the discussed device in 1:1 scale. Results on heat flux, heat capacity and mass flow are easily scalable for a real device with given area of thermal storage cross-section.

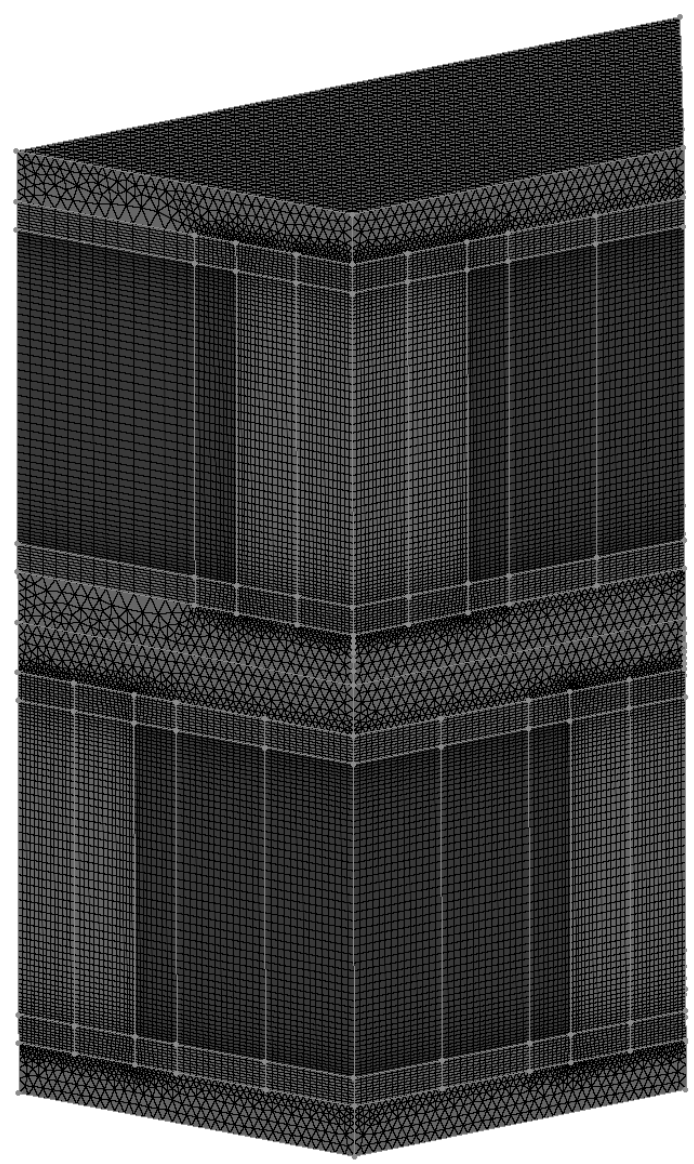

Fig. 6. Overview of fine mesh on Basic Geometry cell

\subsection{Mesh sizing and quality}

Initial meshes considered for this study were created for high resolution fluid boundary layer with $\mathrm{y}^{+}$parameter calculated to value of 5 , based on reference velocity and hydraulic diameter of the bed holes. Real value of $y+$ based on flow-field values is in order of magnitude of 2 , ranging up to 4 where velocity magnitude increases locally. Full 32 layers of fine mesh result in around 14.2 million elements. Estimated physical flow time for completing a single charge/discharge cycle was 6 hours. Completing this physical flow on a 120 core HPC cluster was estimated to be completed in 32 years for a single charge/discharge cycle. Although the quality of the results was superior to the coarse grid, the computational wall-time made the calculation inherently impractical.

For coarse mesh element count was reduced by half in each direction of the mesh for both structured and unstructured domains. This resulted in a mesh with $\mathrm{y}^{+}$ parameter in order of magnitude of 50. Cell count is also drastically reduced to 2 million cells. Real $\mathrm{y}+$ values calculated from flow data range from 13 to 23 where local velocity magnitude is increased. Using a coarsened mesh resulted in achieving relatively practical wall-time with three charge/discharge cycles completed in 21 days.

Mesh quality is comparable for both meshes. Quality figures for full 32 layer mesh are presented in figures 7 thru 9 .

Table 1. Element count per basic geometry.

\begin{tabular}{|c|r|r|}
\hline Element type & Fine grid & Coarse grid \\
\hline Hexa & 439264 & 47774 \\
\hline Tet & 398443 & 74133 \\
\hline Pyramid & 11872 & 2984 \\
\hline Total & 849579 & 124861 \\
\hline
\end{tabular}

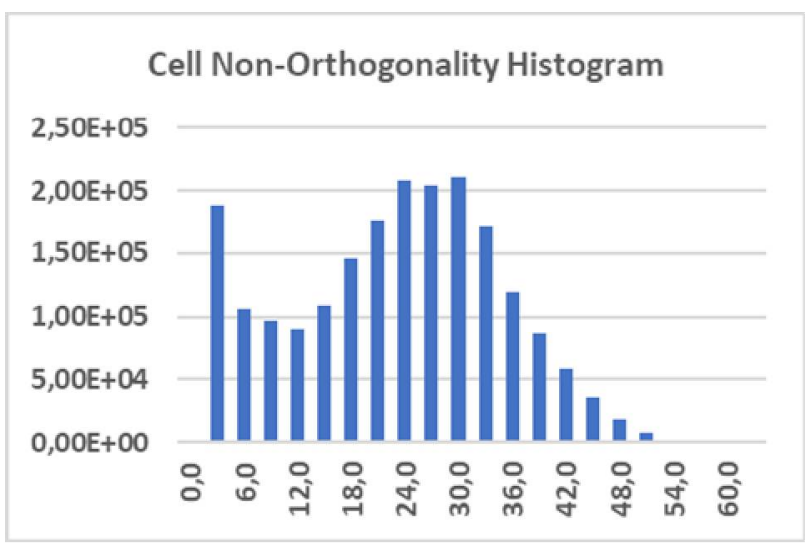

Fig. 7. Coarse mesh cell non-orthogonality histogram. Lower values - better mesh

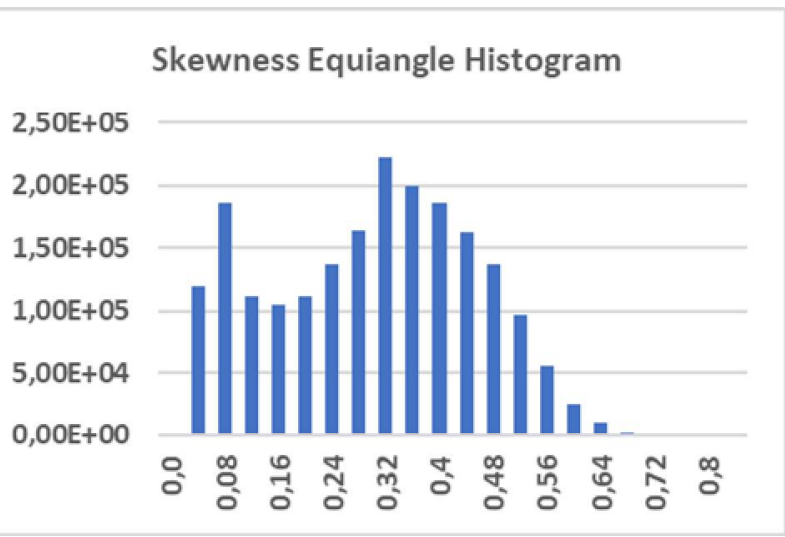

Fig. 8. Coarse mesh cell skewness equiangle histogram. Lower values - better mesh 


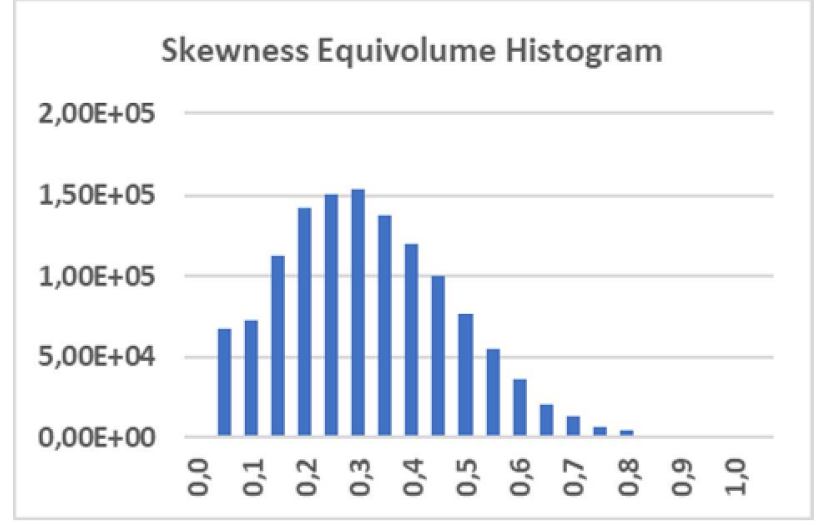

Fig. 9. Coarse mesh cell equivolume histogram. Lower values - better mesh

\section{Results and discussion}

Mesh sizing in the boundary region for conjugate heat transfer require $\mathrm{y}+$ wall sizing in range of 1 . This wall sizing ensures accurate resolution of temperature field and resulting heat flux.

Mesh influence ne the results was observed for both meshes. Heat flux towards the solid body domains was measured during first 50 seconds of flowtime. Although total heat flux towards the solid body domains was comparable for two meshes, with difference of $0,3 \%$ lower heat flux on the coarser mesh, comparison of the first 5 layers showed underestimation of heat flux in range of $10 \%$ for coarser mesh in comparison to fine mesh. As seen on fig 10. fluid temperature drop is more observable on fine mesh in comparison to coarse mesh.

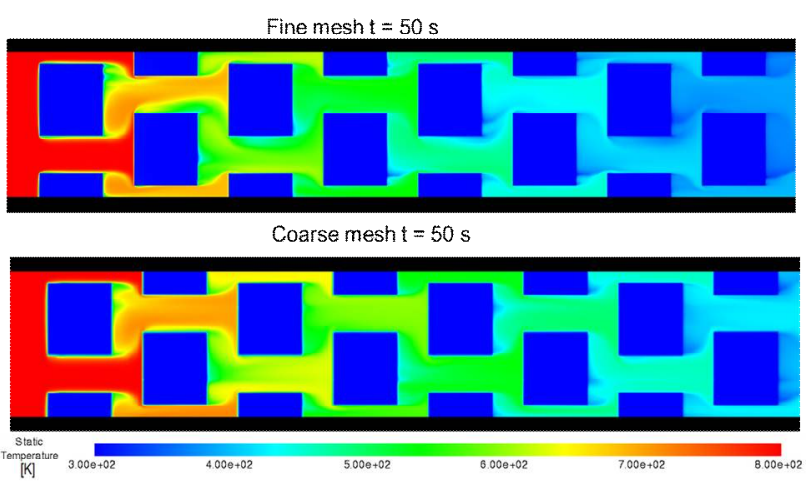

Fig. 10. Overview of fine mesh on Basic Geometry cell

Coarser mesh resulted in better convergence and stabilisation of the calculations. Some of the small scale phenomena were filtered out resulting in more uniform flow field. More details on the numerical approach and results of the calculations are presented in [5].

\section{Conclusions}

It is concluded, that for best quality results, mesh sizing must retain $\mathrm{y}+$ sizing in order of magnitude of 1 for best conjugate heat transfer resolution. However for large scale computations, even implementing a section of the storage requires very large computational resources.
It is suggested that for large scale computations, one should perform a detailed analysis of a single heat storage cell (such as the basic geometry, fig. 3) in order to obtain values of heat capacity of such cell per unit volume and unit area, followed by the implementation of these values in properties of porous material. Further work is described in [6]

This research was supported in part by PLGrid Infrastructure.

\section{References}

1. R. A. Betts, C. D. Jones, J. R. Knight, R. F. Keeling, J. J Kennedy, Nature Climate Change, Nature Research, 6(9), 806. (2016)

2. European Commission, Roadmap 2050. Policy. (2012)

3. J. D. McTigue, A. J. White, C. N. Markides, Applied Energy, 137, 800 (2015)

4. A. Smallbone, V. Jülch, R. Wardle, A. P. Roskilly, Energy Conversion and Management, 152, 221. (2017)

5. B. Ziegler, J. Mosiężny, P. Czyżewski, Int. J. Numerical Methods for Heat \& Fluid Flow. (to be published)

6. J. Mosiężny, B. Ziegler, Int. J. Numerical Methods for Heat \& Fluid Flow. (to be published) 